\title{
A SURFACE-PROJECTION MMI FOR THE FUSION OF BRAIN MR AND SPECT IMAGES
}

\author{
Jiann-Der Lee, Bo-Rong Huang, Chung-Hsien Huang \\ Department of Electrical Engineering,Chang Gung University, Tao-Yuan, Taiwan
}

\begin{abstract}
Recently, maximization mutual information (MMI) of image intensities has been proposed as a new matching criterion for automated multimodality image registration. However, the success of the MMI relies on the similarity of the histogram distribution between the images to be fused. This condition is usually hard to be achieved in practical application. Besides, MMI is time consuming because it needs to find an optimal solution about six parameters (three for shifts and three for rotations) during the registration process. To improve the performance of traditional MMI, a novel scheme named Surface-Projection MMI algorithm (SP-MMI) is proposed here. SP-MMI is a twostage registration algorithm included MMI of surface projection and the following MMI of axial plane. The experimental results, using MR and SPECT images, to confirm the good performance of the proposed method in comparison with the traditional MMI method are also included.
\end{abstract}

Biomed Eng Appl Basis Comm, 2006(August); 18: 202-206.

Keywords: image registration; surface-projection maximization mutual information; CT image; SPECT image; stereotactic surgery

\section{INTRODUCTION}

In clinical applications, medical images obtained from various modalities usually provide valuable information of the patient for diagnosis. For example, functional images (PET, SPECT) provide metabolic or neuro-chemical changes characteristic, and anatomical images (CT, MRI) offer geometric and structural properties. SPECT image is a nuclear medicine technique in which temporal changes in the concentrations of radioactive tracers are recorded in brain and other target organs. It is a non-invasive nuclear medicine technique that utilizes radiopharmaceuticals and a tomography instrument to generate a quantitative image set. This functional

Received: March 1, 2006; Accepted: June 13, 2006

Correspondence: Jiann-Der Lee, Professor

Department of Electrical Engineering,Chang Gung University, Tao-Yuan, Taiwan

E-mail: jdlee@mail.cgu.edu.tw imaging method is firmly established as important scientific and clinical research tool.

Image fusion is a synthesized process, which combined multi-dimension information and displayed on a particular space, with the aim of providing either more condensed or more pertinent information [1]. In general, using MMI [2-9] to register functional and anatomical image, physicians can get more diagnostic information on the focal part. However, it is noted that the image intensity of MR corresponds to the hydrogen density of the body, and SPECT imaging corresponds to radioactive intensity but brain anatomical structure. Their histogram distributions are quite different, and this characteristic will cause MMI fall into a local maximum and result in the system failure [10-11]. Clearly, it is mainly because traditional MMI has high correspondence with the joint MR-SPECT histogram. Moreover, MMI is also a time-consuming algorithm since it needs to derive an optimal solution for three translations and three rotations while registering two modality's spaces. 
In this approach, we present a Surface-Projection MMI (SPMMI) algorithm to improve the performance of the traditional MMI registration. This method can be performed easily and quickly. In addition, the experimental results show that this registration method for MR and SPECT images is as accurate as traditional MMI.

The remainder of this paper is organized as follow. Section 2 presents the proposed method for registration of MR and SPECT images using SPMMI algorithm. In this section, the two stages of SPMMI algorithm are described in details. The experimental results using this approach are presented in section 3. At last, the conclusion is included in final section

\section{THE PROPOSED METHOD}

The whole flowchart of the SP-MMI scheme is illustrated in Fig. 1. SP-MMI is a two-stage method, which includes MMI of surface projection and MMI of axial plane. There are six parameters (three translations and three rotations) needing to be calculated in the process of image registration. But to simplify this problem, we only consider three translation parameters and assume that the rotations are small or nearly zero.

First, surface projection is used to find the 2-D front-face image of each image set. The aim of this stage is to reduce a 3-D volume data to a 2-D image data. Relied on MMI, the surface-projection images of input data sets obtained in axial and coronal direction are aligned by each other to derive the required two translation parameters.

Next, we adjust the shift of the axial plane in sagittal direction and calculate the corresponding MMI at each movement. In this stage, we can obtain the third translation parameter. Finally, the registered data sets are fused and displayed together at the same

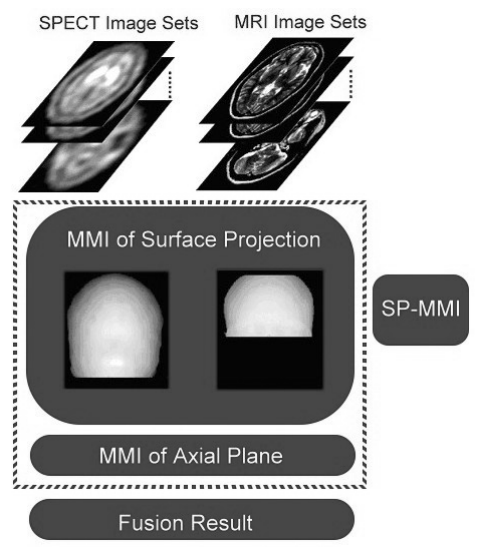

Fig. 1. The flowchart of the SPMMI. coordinate space. Since this registration method combined these two steps described above, it can reduce the computing cost and accomplish the goal of fusion of MR and SPECT accurately. The details of SPMMI are described as below.

\subsection{Maximum Mutual Information}

Maximum Mutual Information (MMI) is developed from information theory, that is applied in the context of image registration to measure the amount of information that one image contains about the other. It is usually used for a measure of similarity for image registration, and belongs to voxel similarity based method. When images are registering, one image is nailed, and the other is shifted, translated, or scaled by a transformation.

The MI measure in the two images is maximized at registration and it is based on entropy, which can be considered to describe the histogram dispersion of the image pixels' intensity. Usually, MI is defined as Eq. (1).

$$
M I(A ; B)=H(A)+H(B)-H(A ; B)
$$

where $H(A)$ and $H(B)$ are the entropies of the images $A$ and $B$, and $H(A ; B)$ is their joint entropy. The joint entropy is given by

$$
H(A ; B)=-\sum_{a=1}^{N} \sum_{b=1}^{N} p(a ; b) \log p(a ; b)
$$

$p(a ; b)$ is the probability of the co-occurrence of gray value $a$ in image $A$ and gray value $b$ in image $B$ at the same coordinate positions, named the joint probability distribution. When the images are aligned, the joint probability should be less than the case of any misalignment.

\subsection{The First Stage: MMI of Surface Projection}

A 3-D volume data can be regarded as a stack of 2-D images, but different kinds of medical images may have different gap size between slice and slice. First, we interpolate the source data sets with an interloation algorithm by cubic spline to normalize the input data in which each voxel is a regular cube as $1 \mathrm{~mm}^{3}$.

The surface projection, as show as in Fig. 2, is a process that projects the facial curve onto a screen. The projective information of this screen changes from distance of space to gray level of image. Fig. 3(a) is the surface projection of SPECT and Fig. 3(b) is the surface projection of MRI, respectively.

Then, moving one image set along the axial direction and coronal direction between reasonable ranges, the value of $\mathrm{MI}$ is calculated at each movement and recorded. Therefore, a pair of the axial and coronal 


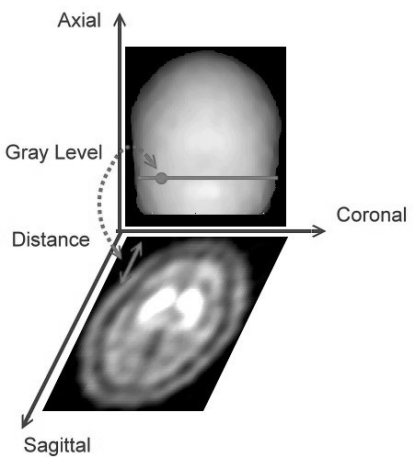

Fig. 2. The process of surface projection.

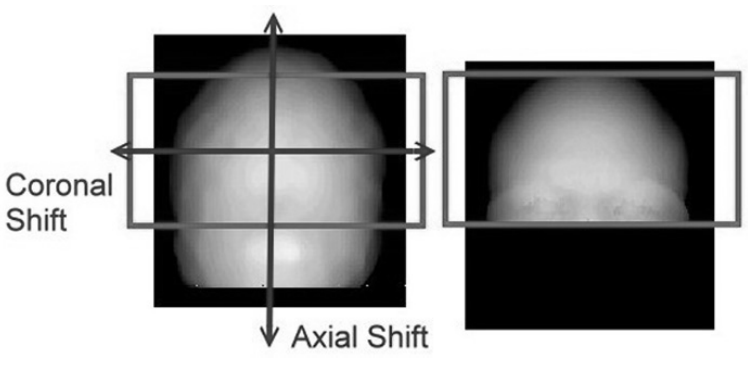

(a)SPECT

(b)MRI

Fig. 3. Facial images ' registration by the surface projection.

translations with the maximum value of MI is considered as the optimal translations in this stage and will be passed to next stage.

\subsection{The Second Stage: MMI of Axial Plane}

In the stage, we adjust the shift of the axial plane in sagittal direction. In other words, two axial plane images at the same position of both data sets are selected, and are calculated by the MMI with both of them. As show as in Fig. 4, the only translation needed to be changed at this step is used for the sagittal direction. Adjusting during a suitable range and calculating the value of MI at each movement can help to find the best translation of sagittal direction. Finally, three registration parameters of translation can be obtained by using the processes described above.

\section{EXPERIMENTAL RESULTS}

In the experiments, MR images used are T2weighted image with a TR of $4000 \mathrm{msec}$ and TE of 90

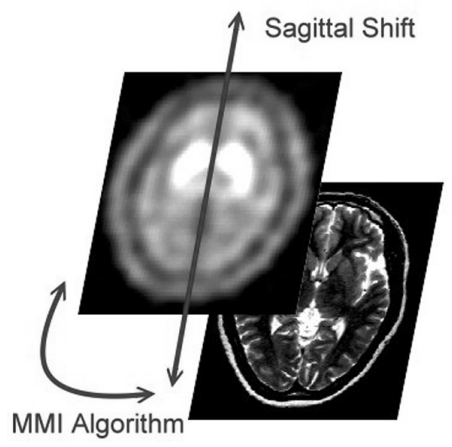

Fig. 4. MMI of axial plane.

msec, slice thickness is $3 \mathrm{~mm}$, and image's matrix size is $256 \times 256 \times 36$ voxels. And SPECT images are Tc$99 \mathrm{~m}$ labeled tropanes of TRODAT, $128 \times 128 \times 64$ voxels, and each voxel is a $2.9 \mathrm{~mm}^{3}$ cubic. In our case, the fused images can aid physicians to analyze the quantity contained of dopamine in putamen and caudate nucleus for the diagnosis of Parkinsion's disease. In fact, this scheme is not only useful for Parkinson' s disease, but also Alzheimer's disease, epilepsy or other brain degenerative diseases rely on MR and SPECT images for clinical diagnosis, evaluation, and treatment.

To compare the performance of the proposed SPMMI and traditional MMI, three affined-invariants including area, long-axis, and short-axis are employed to calculate error functions of both slice set of joint object.

Three error functions in terms of area, long-axis and short-axis are listed in Eqs. (3)-(5):

$$
\begin{gathered}
e_{\text {area }}=\frac{1}{S} \sum_{s_{i}}^{S}\left|\frac{\operatorname{area}\left(a_{s_{i}}\right)}{\max (\operatorname{area}(a))}-\frac{\operatorname{area}\left(b_{s_{i}}\right)}{\max (\operatorname{area}(b))}\right| \\
e_{\text {long }}=\frac{1}{S} \sum_{s_{i}}^{S}\left|\frac{\operatorname{long}\left(a_{s_{i}}\right)}{\max (\operatorname{long}(a))}-\frac{\operatorname{long}\left(b_{s_{i}}\right)}{\max (\operatorname{long}(b))}\right| \\
e_{\text {short }}=\frac{1}{S} \sum_{s_{i}}^{S}\left|\frac{\operatorname{short}\left(a_{s_{i}}\right)}{\max (\operatorname{short}(a))}-\frac{\operatorname{short}\left(b_{s_{i}}\right)}{\max (\operatorname{short}(b))}\right|
\end{gathered}
$$

where $S$ is total slice number of joint, $s_{i}$ is the slice sequence of image sets $\boldsymbol{a}$ and $\boldsymbol{b} \cdot \boldsymbol{m a x}()$ denotes the maximum value of whole image set. And area(), long(), and short() represent values of area, long-axis, and short-axis, respectively.

Compared with the traditional MMI, in the term of calculation time for finding the three translation parameters in a $C \times A \times S$ solution space, the traditional MMI needs to calculate the MMI $(C \times A \times$ 
$S)$ times, while SP-MMI needs to calculate it $(C \times$ $A+S)$ times. From Fig. 5, it is observed that the error functions of SP-MMI are close to traditional MMI totally. That is, the proposed scheme is simple and fast, but still possesses the accuracy as well as traditional MMI in the registration process.

Besides, Fig.6 shows the fused 2-D images. (a) is fused by conjunctive operator, (b) is fused by disjunctive operator, (c) is fused by compromise-like operator [12], and (d) is the fused result by pasting the ROI of SPECT onto the MRI. Fig.7 is the fused result displayed in 3-D form.

\section{CONCLUSIONS}

In this approach, we have proposed a two-stage image fusion scheme named SP-MMI to solve the fusion problem of MR and SPCET images. This scheme divides the registration process into two stages,

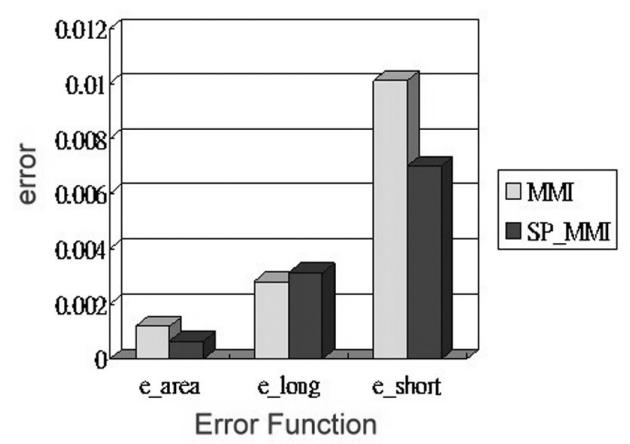

Fig. 5. The error functions of SP-MMI and traditional MMI.

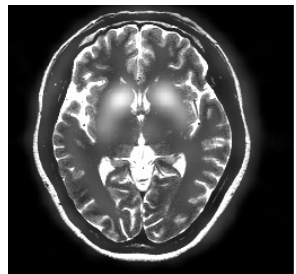

(a)

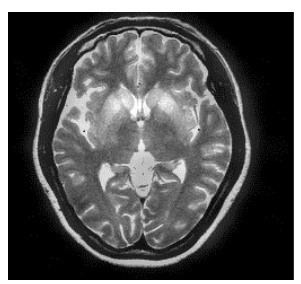

(c)

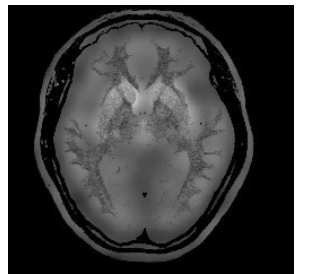

(b)

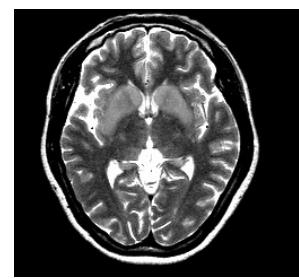

(d)
Fig. 6. The 2-D and 3-D display of the fused image.

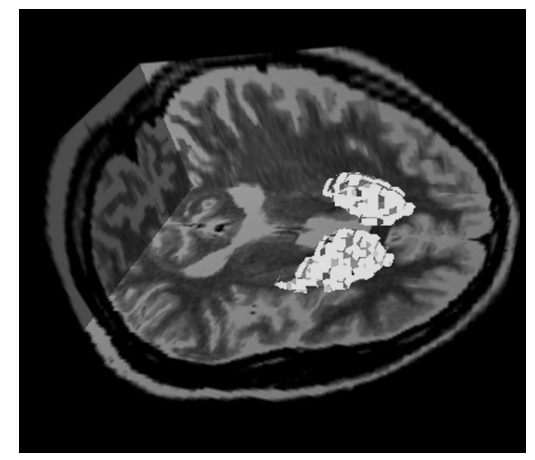

Fig. 7. The 3-D display of the fused image.

i.e., MMI of surface projection and MMI of axial plane, and can be performed easily and quickly than traditional MMI.

The success of SP-MMI is under the reasonable assumption that the source data sets have small or near zero rotation for three orthogonal axes. The experimental results, using MR and SPECT images, to confirm the good performance of the proposed method in comparison with the traditional MMI method are also presented.

\section{ACKNOWLEDGMENT}

The authors would like to thank the National Science Council of the R.O.C. for financial support of this manuscript under contract No. NSC94-2614-E182-002

\section{REFERENCES}

1. J.B. A. Maintz and M.A Viergever, "A survey of medical image registration" , Medical Image Analysis, 1998; 2:1-36.

2. F. Maes, D. Vandermelen, and P.Suetens, "Medical Image Registration Using Mutual Information" , Proceedings of the IEEE, 2003; 91:1699-1722.

3. P. Viola, W. Wells," Alignment by maximization of Mutual Information," Proceedings of the fifth International Conference on Computer Vision, 1995: 15-25.

4. A. Collignon, F. Maes, D. Delaere, D. Vandermeulen, P. Suetens, and G. Marchal, "Automated multimodality image registration using information theory", Infromation Processing in Medical Imaging, 1995: 263-274.

5. F. Maes, A. Collignon, D. Vandermelen, G. Marchal, and P. Suetens, "Multi-modality image registration by maximization of mutual information," IEEE Trans. Med. Imaging 16, 1997: 187-198. 
6. C. R. Meyer, J. L. Boes, B. Kim, P. H. Blad.,R. Zasadny, P. V. Kison, K. Koral, K. A. Frey, R. L. Wahl, "Demonstration on accuracy and clinical versatility of mutual information for automatic multi-modality image fusion using affine and thinplate spline warped geometric deformations, Med. Image Analysis, vol. 1, 1997; 1: 195-206.

7. J. B. A. Maintz, E. W. H. Meijering, M. A. Viergever, "general multimodal elastic registration based on mutual information" , Medical Imaging, SPIE Press, vol. 1998; 3338: 144-145.

8. T. Gaens, F. Maes, D. Vandermelen, P. Suetens, Non-rigid multimodal image registration using mutual information", Medical image computing and computer-assisted intervention, Lecture Notes in Computer Science, 1998; 1496: 1099-1106.

9. W. M. Wells III, P. Viola, and R. Kikinis, "Multimodal volume registration by maximization of mutual information" , Med. Image Analysis, 1997; 1: 35-51.

10. H. Chen, and P.K.Varshney, "Mutual informationbased CT-MR brain image registration using generalized partial volume joint histogram estimation” , IEEE Trans. Medical Imaging, 2003; 22: 1111-1119.

11. R. He, P. A. Narayana, "Global optimization of mutual information: application to threedimensional retrospective registration of magnetic resonance images" , Computerized Medical Imaging and Graphics, 2002; 26: 277-292.

12. I. Bloch, "Information combination operators for data fusion: a comparative review with classification”, IEEE Trans. Syst. Man. Cybern, 1996; 26: 52-67. 